\title{
Métodos Empíricos em Pesquisa do Envelhecimento entre Minorias: um caso para triangulação sociológica
}

KATE G. PRIGKETT" E JAGQUELINE L. ANGEL

\section{Resumo}

A triangulação, há muito, vem sendo empregada para validar estudos cujos resultados são alvo de críticas por basearem-se em amostras muito pequenas ou por não permitirem generalização. Com um foco especial no uso de múltiplas fontes de dados quantitativos, expandimos o uso da triangulação em um contexto sociológico, argumentando que não só a sociologia é um campo apto ao uso da combinação de diferentes bases de dados, mas que esta é uma ferramenta útil ao se analisarem populações de difícil acesso e menos favorecidas. Destacando exemplos de múltiplas bases de dados empregadas usualmente para explorar questões sobre o envelhecimento de pessoas de origem latino-americana nos Estados Unidos, discutimos como estas distintas fontes podem ser aplicadas para fortalecer os resultados. Concluímos, discutindo a aplicação da triangulação como um meio de tornar as análises sociológicas acessíveis e relevantes para o campo das políticas, o que acreditamos ser um esforço necessário para assegurar uma perspectiva sociológica no âmbito mais amplo da discussão de políticas.

Palavras-chave: Triangulação. Métodos mistos em sociologia. Minorias. Idosos latino-americanos.

\footnotetext{
*Assistente de Pesquisa - Departamento de Sociologia, The Population Research Center, University of Texas, Austin (Estados Unidos) E-mail: kate.prickett@utexas.edu.

${ }^{* *}$ Professora de Sociologia e Políticas Públicas, Departamento de Sociologia, Professora Associada da Lyndon B. Johnson School of Public Affairs e do Population Research Center - University of Texas, Austin (Estados Unidos). E-mail: jangel@austin.utexas.edu.
} 


\section{Empirical Methods in Minority Aging Research: The Case for Sociological Triangulation ${ }^{1}$}

\section{Abstract}

Triangulation has long been employed to provide validation for study findings that are easily critiqued because of small sample sizes or lack of generalizability. With a particular focus on using multiple quantitative data sources, we expand the use of triangulation within a sociological context, arguing that not only is sociology a field ripe for the use of combining different datasets, but that it is a useful tool when examining hard-to-reach and disadvantaged populations. Highlighting examples from multiple datasets that are typically employed to explore issues of Hispanic aging in the United States, we discuss how these different sources can be deployed to leverage findings. We conclude by discussing the application of triangulation to make sociological analyses policy accessible and relevant, a necessary pursuit, we argue, to ensure a sociological voice is part of the broader policy discussion.

Keywords: Triangulation. Mixed methods in sociology. Minorities. Hispanic aging.

\section{Introdução}

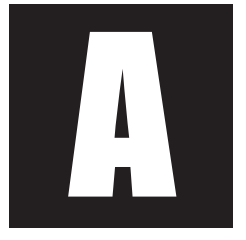

triangulação, há muito, vem sendo empregada para validar estudos cujos resultados são alvo de críticas por utilizarem amostras muito pequenas ou por não permitirem generalização. Isto ocorre com frequência na investigação de populações de difícil acesso ou em estudos baseados, principalmente, em dados qualitativamente ricos e muito detalhados. Contudo, conforme já destacado por outros autores, e como iremos desenvolver neste estudo, a triangulação também pode ser uma ferramenta para expandir e aprofundar nossa compreensão da sociologia do envelhecimento.

\footnotetext{
${ }_{1}^{1}$ Tradução de Liana Fernandes.
} 
Além de trazer exemplos de usos tradicionais da triangulação, justificativas para o uso desta técnica, bem como suas limitações e ciladas, discutiremos de que forma ela pode ser usada para tornar a pesquisa sociológica relevante para o campo das políticas. Examinaremos, por exemplo, como a triangulação pode ser usada para problematizar questões de pesquisa e para criar gradações para estatísticas de nível populacional, de modo a tornar os resultados relevantes para a discussão de políticas. Sustentamos, de fato, que é por sua capacidade de traduzir uma pesquisa acadêmica em contextos de políticas, que a triangulação, através do uso de dados quantitativos, pode trazer os maiores avanços.

Discutimos estas questões, utilizando exemplos de diversos e complexos levantamentos (surveys), geralmente aplicados em pesquisas sobre a população idosa de origem latino-americana nos EUA - uma minoria que, de um modo geral, é de difícil acesso. Além de fornecer informações sobre conteúdo, benefícios e desvantagens das bases de dados comumente empregadas na análise dessa população, usamos pesquisas empíricas atuais para ilustrar como múltiplas fontes de dados podem ser aproveitadas para examinar o envelhecimento da população de origem latino-americana e de outras populações americanas minoritárias. Pesquisas estas que, muitas vezes, se deparam com situações que envolvem escopo limitado de variáveis e amostras de tamanho reduzido.

\section{A Sociologia da Triangulação}

O convite aos sociólogos para adotar a triangulação como forma de análise não é novidade. Em 1969, Smelser apontou a sociologia como uma disciplina apta à combinação de abordagens metodológicas, afirmando que a sociologia, em comparação a outras ciências sociais, não possui um marco conceitual único universalmente aceito. Contudo, ao 
invés de identificar a sociologia como um abismo metodológico sem embasamento, ele destacou a oportunidade para que diferentes marcos teóricometodológicos contribuam com a iniciativa sociológica (Smelser, 1969). Embora Smelser limite boa parte de sua discussão aos marcos conceituais, uma disciplina que recorra a um conjunto variado de teorias é, também, boa candidata à aplicação de uma variedade de metodologias. Esta abordagem, chamada de "articulação complementar", constitui, então, uma ferramenta necessária para a exploração de questões sociológicas, particularmente na medida em que aproveita dados coletados de fontes multidisciplinares, criando um quadro mais holístico das vidas na sociedade.

A triangulação é um método abrangente e diversificado para efetivar a articulação complementar de Smelser. Denzin (1978) define quatro tipos básicos de triangulação: 1) de dados, 2) de investigador, 3) de teoria e 4) metodológica. Embora todos estes tenham um papel em métodos sociológicos aplicados, iremos focar a primeira forma de triangulação de dados - para explorar de que forma diferentes fontes de dados podem ser aproveitadas para criar um quadro mais holístico de populações pouco estudadas ou difíceis de acessar. Frequentemente chamadas de "populações especiais," estes grupos geralmente apresentam problemas difíceis de tratar, doenças cujo tratamento é caro, e/ou dispõem de recursos escassos. Por exemplo, pessoas mais velhas, mulheres grávidas e pessoas portadoras de deficiências têm necessidades ampliadas de serviços de saúde. Da mesma forma, pessoas com doenças como HIV/AIDS ou distúrbios mentais precisam de serviços de saúde especializados. E, finalmente, populações com recursos escassos, ou socialmente excluídas, como populações de baixa renda ou indígenas, também são consideradas especiais. Estas populações são muito heterogêneas, fazendo da triangulação, muitas vezes, uma ferramenta necessária para examinar diferenças internas aos grupos, já que uma única base de dados normalmente não dispõe dos recursos para examinar esse nível de diversidade. 
A triangulação de diferentes fontes de dados é uma forma importante de criar um quadro mais abrangente da realidade social de um grupo. Muitos sociólogos (particularmente os que utilizam métodos quantitativos) usam dados secundários que, não raro, são coletados com base em outro marco conceitual ou com base em uma questão de pesquisa diferente da explorada pelo usuário secundário. Nesse sentido, incorporar diferentes fontes de dados permite aos pesquisadores utilizar variáveis coletadas em um estudo para complementar a análise de outra fonte de dados, a qual pode não ter à disposição todo o escopo de variáveis necessárias para a abordagem da pesquisa proposta. Por exemplo, um conjunto exaustivo de dados epidemiológicos pode não conter informações detalhadas sobre constructos sociológicos como a organização familiar. Embora o estudo epidemiológico possa fornecer detalhes minuciosos sobre a necessidade por cuidados de indivíduos, através de informações sobre sua saúde e incapacidades, pode haver poucos dados sobre os apoios sociais a que eles têm acesso, tais como familiares ou cuidadores em sua região. Uma fonte de dados secundários, como informações censitárias sobre composição familiar, pode ser utilizada para fornecer um instantâneo das vidas de populações similares à amostra em estudo e de seu acesso à rede de apoio social.

No entanto, mesmo em estudos em que o pesquisador esteja aplicando apenas uma fonte de dados, pode não haver, na base dos dados longitudinais, a disponibilidade de dados consistentes, que correspondam às necessidades de uma abordagem metodológica específica. Por exemplo, pesquisadores que usam dados em painel podem não obter as mesmas medidas em cada série de dados. Se o pesquisador estiver usando análise de séries históricas ou modelos de efeitos fixos para avaliar mudanças ao longo do tempo, ele não poderá utilizar uma determinada medida como, por exemplo, uma variável invariante no tempo. Nesse sentido, a seleção de métodos pode, com frequência, despertar a necessidade de triangula- 
ção de dados. Por exemplo, utilizando análise de séries históricas, exploramos o papel desempenhado por deficiências e por recursos de capital humano para explicar a transição do viver sozinho ao viver com a família, entre idosos de origem mexicana nos EUA. Ademais, analisamos como o momento dessa transição se relaciona com a mortalidade e descobrimos que, para essa população, a transição para um arranjo de vida em família está também altamente correlacionada com a hora de morrer (Prickett; Angel, 2012). Um dos interesses desse estudo é determinar a confiança que idosos americanos de origem mexicana depositam na família, com relação aos cuidados no final da vida. Contudo, uma sondagem suplementar da família do idoso entrevistado somente é coletada durante uma parte do estudo. Ao mesmo tempo em que aproveitamos a estrutura do estudo longitudinal para observar trajetórias de arranjos de vida, fizemos uso de variáveis fixas no tempo sobre a responsabilidade dos cuidadores para contextualizar nossos achados principais.

A triangulação é empregada, às vezes, não para contextualizar a análise, mas para dar suporte ou testar a robustez de um achado importante. Esta é uma questão especialmente importante ao se lidar com populações minoritárias vulneráveis. Por exemplo, um coeficiente pouco significativo pode ser vítima da força estatística, e não um resultado efetivamente nulo. Enquanto a base de dados principal empregada pode ter sido necessária, em vista de sua gama diversa de variáveis, achados específicos podem ser reforçados com o uso de uma base de dados secundária, que contemple uma amostra de tamanho maior (tais como dados censitários ou sondagens de saúde da população, às quais falta uma gama de variáveis), como forma de dar suporte a achados estatisticamente fracos. De modo semelhante, uma descoberta atípica e inesperada pode ser apoiada ou moderada pelo uso da triangulação. Por exemplo, um conjunto de dados suplementar pode ser usado para demonstrar que é 
difícil replicar um achado contraintuitivo com uma amostra diferente. Ou, alternativamente, uma segunda ou terceira fonte de dados pode orientar hipóteses sobre o porquê dos resultados alcançados não serem, afinal, tão surpreendentes.

\section{Dados sobre População de Origem Hispânica nos Estados Unidos}

Como já foi mencionado, para conduzir análises sobre a população de idosos de origem latino-americana nos EUA, não raro é necessária mais de uma base de dados para o estudo da saúde e status funcional, já que a maioria delas apresenta limitações, que só podem ser superadas pela combinação com outras. Com relação especificamente ao desenho de modelos dinâmicos em painel, para idosos de origem latino-americana nos EUA, pode ser necessário valer-se de fontes de dados diferentes, por questões de índices de migração relativamente maiores entre idosos latino-americanos em comparação a idosos brancos não latino-americanos e afro-americanos. Para imigrações de mexicanos de mais idade nos EUA, por exemplo, o retorno ao México como resultado de doença ou necessidade de cuidados da família (Palloni; Arias, 2004). O efeito 'regresso de migrantes' tem impacto considerável nas estimativas de morbidade e mortalidade.

Esses dados consistem de dois tipos de sondagens: (1) as que fornecem informações sobre saúde e capacidade funcional, assim como alguma informação sobre apoio social; e (2) aqueles que fornecem informações de base populacional sobre diferenças entre grupos na distribuição de fatores de riscos de saúde, como baixa escolaridade e pobreza. Como já dito, pesquisadores, por necessidade, podem utilizar esse procedimento, já que nenhuma base de dados proporciona informações detalhadas tanto de saúde quanto de riscos econômicos e sociais para a saúde para 
grandes populações de idosos americanos de origem mexicana. As seguintes bases de dados permitem a investigadores fazer inferências a respeito do impacto de fatores como assimilação econômica e cultural, família e recursos sobre a saúde e a capacidade funcional e seu impacto para a utilização de serviços de saúde entre idosos de origem mexicana nos EUA. O que se vai apresentar é uma seleção, não uma lista exaustiva das bases de dados geralmente empregadas no estudo de idosos de origem latino-americana nos EUA.

\subsection{Microdados de Utilização Pública}

para pessoas de 60 ou mais- (PUMS-O)

O PUMS-O (Public Use Microdata for persons 60 years and over) consiste de uma amostra de três por cento dos indivíduos de 60 anos ou mais, e inclui informações disponíveis nos arquivos padrão da base de microdados de uso público do Departamento de Recenseamento dos EUA (Census Bureau). Esses dados podem ser combinados com os PUMS padrão de cinco por cento ou de um por cento, para ampliar a amostra de idosos de origem latino americana, especialmente daqueles em idade muito avançada. O arquivo de 1990 está estratificado por idade e consiste de subamostras de três faixas etárias amplas: 60-74; 75-89, e 90 anos ou mais. Uma das vantagens especiais desses dados é que a disponibilidade de um grande número de casos permite uma análise detalhada dos idosos em idade mais avançada, e são ideais para comparação de subgrupos específicos da população de origem latino-americana. Esses dados podem ser usados para examinar várias questões de pesquisa, inclusive diferenças na institucionalização, desagregadas por idade e por sexo para diferentes grupos; diferenças entre grupos quanto à capacidade funcional dos que vivem com outros; diferenças nos modos de vida e migração após a aposentadoria; diferenças em equidade familiar e participação na força de trabalho; diferenças entre grupos quanto às fontes de renda; e muito mais 
(Angel; Hogan, 1992). Os dados incluem indicadores de ordem cultural como cidadania, origem étnica, ano de chegada aos Estados Unidos, histórico de migração, país natal, idioma falado em casa e proficiência em língua inglesa. Também há informações disponíveis sobre limitações de mobilidade e com relação aos cuidados pessoais.

O PUMS-O contém códigos que identificam "gerências distritais" identificadas pelas secretarias de planejamento em saúde dos estados. Esses identificadores possibilitam, em certos casos, anexar ao arquivo informações sobre a área dos serviços e ter controle sobre fatores contextuais. Como parte do próprio estudo, investigaremos a possibilidade de usar estes e outros identificadores geográficos para monitorar características socioeconômicas e de prestação dos serviços de saúde em cada localidade.

\subsection{Populações Hispânicas Estabelecidas}

para o Estudo Epidemiológico dos Idosos (H-EPESE)

O estudo longitudinal de saúde do idoso americano de origem mexicana (Longitudinal Study of Mexican American Elderly Health - Hispanic Established Populations for Epidemiological Studies of the Elderly ou H-EPESE) consiste de uma amostra representativa de 3.050 cidadãos americanos de origem mexicana, com idade de 65 anos ou mais, que residem em cinco estados do sudoeste: Texas, Califórnia, Novo México, Arizona, e Colorado (Markides; Rudkin; Angel; Espino, 1997). O National Institute on Aging fundou o H-EPESE em 1992. Este é um projeto bastante diferenciado, pois foi o primeiro levantamento epidemiológico longitudinal de idosos de origem mexicana dos EUA e, como tal, constitui um estudo de referência sobre a saúde e as transições nos modos de vida dessa população. O levantamento inicial foi conduzido entre 1993 e 1994. Seis entrevistas adicionais de acompanhamento foram conduzidas, com a última série de dados sendo coletada entre 2010-2011. Esta composição da amostra (1) nos dá a neces- 
sária robustez estatística para obter estimativas estáveis relativas a pequenos subgrupos da população idosa de origem mexicana, (2) compensa as perdas ao longo de dois anos da entrevista, e (3) produz uma amostra representativa dos idosos de origem mexicana do sudoeste americano.

Esse levantamento inclui um histórico detalhado de cada indivíduo pesquisado, bem como informações sobre capacidade funcional, doenças físicas e estado mental. Também estão sendo coletadas informações sobre o acesso do entrevistado a serviços de atenção médica com regularidade, o número de hospitalizações que ele ou ela teve durante o último ano, a razão e o local da última visita ao médico e as fontes de custeio da assistência à saúde. O estudo compreende extensas entrevistas domiciliares, que incluem histórico clínico focado em afecções crônicas, como angina, em doenças cardiovasculares, histórico de derrame cerebral, hipertensão, câncer, diabetes, fraturas, doenças da vesícula biliar, artrite e incontinência. Um dos aspectos singulares desses dados refere-se ao fato de que entrevistadores treinados realizaram avaliações médicas e avaliações de funcionamento físico com base em atividades. Esses dados também fornecem informações demográficas básicas de natividade e migração.

\subsection{Levantamento de idosos de origem latino-americana (The Commonwealth Fund)}

O levantamento de idosos hispânicos (Survey of Elderly Hispanics SEH) consiste de um levantamento telefônico, realizado em 1988 pela Westat, Inc. para o Commonwealth Fund, com 2.299 idosos de origem latino-americana, de 65 anos ou mais (Davis, 1990). Até hoje, esta é uma das melhores fontes de dados disponíveis para avaliar recursos de apoio formal e informal, assim como as necessidades de cuidados de saúde de uma amostra nacional de idosos de origem latino-americana nos Estados Unidos. O estudo consiste de um levantamento telefônico conduzido en- 
tre agosto e outubro de 1988. Diversas medidas foram tomadas para minimizar os problemas associados a um levantamento telefônico de uma população idosa de origem latino-americana. Temos confiança na utilidade desses dados por diversas razões. Primeiro, noventa e dois por cento (92\%) dos domicílios de ascendência latino-americana nos Estados Unidos têm, no mínimo, um telefone (Westat, 1989). Segundo, entrevistadores acostumados a interagir com esse segmento da população foram designados para entrevistar pessoas que, por qualquer razão, haviam se recusado a participar do estudo. Terceiro, pessoas que não responderam ao primeiro contato telefônico foram contatadas ao longo de um período de duas semanas em um total de seis novos contatos (2 dias, 3 noites, 2 finais de semana).

Para assegurar um número razoável de idosos de origem latinoamericana, foram triados 85.404 domicílios, em regiões dos Estados Unidos com altas concentrações de população de origem latino-americana, para identificar domicílios desse segmento populacional com idosos de 65 anos ou mais. A identificação do domicílio como latino-americano foi baseada em etnia auto-declarada. Todos os idosos localizados em domicílios de latino-americanos foram convidados a participar de uma entrevista telefônica extensa. A pesquisa proposta empregará uma sub-amostra de 2.019 idosos americanos de origens mexicana, cubana e porto-riquenha, com 65 ou mais, que tenham completado com êxito a entrevista. Os tamanhos das amostras dos três sub-grupos são os seguintes: origem mexicana (937); origem porto-riquenha (368); e origem cubana (714). As entrevistas foram conduzidas em inglês (13\%) e em espanhol (87\%).

Além de informações clínicas, os entrevistadores bilíngues coletaram informações sobre o modo de vida dos entrevistados idosos, ano de migração para os Estados Unidos, recursos econômicos, benefícios de aposentadoria, rede social, apoio familiar, incapacidade funcional e condições de saúde. O índice geral de resposta ao levantamento foi de 80\%. 


\subsection{Análise Nacional de Saúde e Nutrição - III}

O NHANES-III (National Health and Nutrition Examination SurveyIII) fornece dados sobre fatores de risco para doenças, a partir de uma grande amostra de americanos de origem não latina e de origem mexicana com mais de 60 anos (National Center for Health Statistics, 1994). A primeira fase da sondagem de seis anos coletou dados entre outubro de 1988 e outubro de 1994. Como nas Análises Nacionais de Saúde e Nutrição anteriores, a NHANES-III emprega amostragem probabilística estratificada, multi-estágios, para avaliar as condições de saúde de uma amostra de indivíduos não institucionalizados nos Estados Unidos. Esta é uma das poucas sondagens nacionais de larga escala que coleta uma grande quantidade de informações sobre saúde física. A informação é baseada em histórico clínico, exames laboratoriais, medidas físicas e avaliações de incapacidades. O levantamento inclui "sobreamostras" ${ }^{2}$ dos subgrupos de indivíduos idosos e da população de origem mexicana.

O NHANES-III é um conjunto de dados sem igual, pois contém os resultados de um exame médico minucioso no qual os achados anormais são codificados nos termos de categorias da Classificação Internacional de Doenças (CID), nona revisão. Um exame, conduzido por médico, avaliou todos os principais sistemas fisiológicos, qualificando-os como normais ou anormais. O exame médico foi concluído com uma avaliação geral da condição de saúde do examinado como excelente, muito boa, boa, regular ou ruim. O exame incluiu também medidas de pressão san-

\footnotetext{
${ }^{2}$ No original "oversamples". Técnica utilizada em surveys, que consiste em ampliar o número de entrevistas com um sub-grupo da população pesquisada, com vistas a melhorar a confiabilidade dos resultados para aquele subgrupo. Este procedimento reduz os erros potenciais de amostragem aleatória para o grupo em questão. Evidentemente, na tabulação dos dados para a amostra geral, a sobreamostra é desconsiderada e o subgrupo retorna à sua proporção original (Nota da tradutora).
} 
guínea, altura, peso e relação peso-altura, densidade óssea, impedância do tímpano, audiometria, visão, tolerância à glicose e exames laboratoriais (p. ex., radiografias) para artrite, diabetes e anemia. Além de fazer um diagnóstico, o médico responsável também administrava um teste de rendimento para avaliar o grau total de debilidade da função motora geral.

Além do exame médico, foi obtido um histórico clínico detalhado de cada examinado. As entrevistas incluem perguntas sobre nutrição, atividade física, saúde mental e hábitos de saúde. Também foram coletadas informações sobre o acesso do entrevistado a serviços regulares de cuidado médico, número de hospitalizações que ele ou ela sofreu durante o último ano, a razão e o local da última visita ao médico e suas fontes de custeio de serviços de saúde. Além disso, a sondagem domiciliar reúne informações sobre atividades da vida diária (AVD) e sobre uma série de condições específicas de saúde, como diabetes, cálculos biliares, doenças cardiovasculares, dor no peito ou no abdômen, e problemas dentários.

\section{Implicações para as Políticas Públicas}

Como buscamos demonstrar, essas bases de dados evidenciam que, embora cada uma delas seja importante para o estudo de idosos de origem latino-americana, e constituam esforços notáveis de coleta de dados, empregar apenas uma dessas bases de dados não é suficiente para traduzir a complexidade das vidas dessas pessoas para o cenário das políticas públicas. Nesta seção, iscutiremos como a triangulação pode ser usada para contextualizar a pesquisa para um público de formuladores de políticas. Iremos focar três áreas importantes em que se pode aproveitar a triangulação de fontes de dados. Primeiro, a triangulação pode ser usada para problematizar questões de pesquisa junto a autoridades e formuladores de políticas. Segundo, a triangulação pode ser usada para tornar 
os resultados de pesquisa generalizáveis em nível populacional. E, terceiro, permite aprimorar as estatísticas de nível populacional, contribuindo não somente para explicar tendências de nível macro, mas também para orientar futuras prioridades de pesquisa e financiamento e, inclusive, novos esforços de coleta de dados.

O uso da triangulação para problematizar questões de pesquisa não é um fenômeno recente. Muitos estudos iniciaram com estatísticas de nível populacional sobre o número de pessoas que sofrem de algum tipo de enfermidade ou que são categorizadas com um status social específico. No entanto, o uso de dados de nível populacional para problematizar questões de pesquisa pode ser aprimorado. Os pesquisadores querem definir o número de pessoas que o estudo em nível individual representa dentro de uma população. Este número pode ser estendido, utilizandose dados censitários ou bases de dados amplas, de abrangência nacional, para criar um quadro mais detalhado, não limitado a um parâmetro populacional, mas que examine essas tendências ao longo do tempo em comparação com outros subgrupos populacionais ou com desigualdades internas a grupos. Esta perspectiva ampliada pode gerar um maior senso de urgência para a agenda de pesquisas. Por exemplo, Angel, Herrera, Markides e Torres-Gill (2011) utilizam o Quinto Levantamento Nacional de Participantes do Programa Estatuto do Idoso Americano (Older Americans Act - OAA) (2009) sobre serviços de enfermagem/cuidados de saúde nos EUA para examinar quem está recebendo serviços de apoio. As autoras, então, comparam esses resultados com o Estudo sobre Aposentadoria e Saúde (Health and Retirement Study - HRS) e o H-EPESE, para determinar se os serviços de enfermagem estão atingindo os idosos de origem latino-americana e afro-americanos mais vulneráveis. Da mesma forma, um estudo sobre os beneficiários do programa de nutrição previsto no Título III do Estatuto do Idoso Americano (OAA) usa o Quinto Levantamento 
Nacional de Participantes do Programa Estatuto do Idoso Americano para verificar se esses beneficiários possuem características em comum com os idosos americanos mais vulneráveis à internação em asilos, identificados no Suplemento Social e Econômico Anual relativo ao último Censo Populacional produzido pela Agência de Recenseamento dos EUA e no Estudo sobre Aposentadoria e Saúde (Altshuler; Schimmel, 2010). Esta comparação possibilita que os resultados orientem a interpretação das políticas sobre o papel do programa de nutrição (OAA Title III) para ajudar idosos a envelhecer em seus lares.

Assim como o uso de fontes de dados diferentes pode contribuir para problematizar questões de pesquisa, estatísticas de nível populacional podem ser usadas para tornar os resultados generalizáveis à escala populacional, de modo a influenciar mudanças programáticas e de políticas. A maior parte dos estudos não inclui informações sobre participação em programas voltados a adultos idosos. Como parte de um programa de pesquisa mais amplo sobre mulheres em idade de pré-aposentadoria, Angel, Montez e Angel (2011) exploram desigualdades nos fundos da aposentadoria entre mulheres nesta faixa de idade. Uma pesquisa de acompanhamento foi conduzida durante o debate da reforma do sistema de saúde americano, em 2010. Esta pesquisa utilizou os resultados apresentados sobre desigualdades raciais e étnicas e expandiu essa literatura, usando dados censitários para estimar como o serviço de saúde poderia ser estendido a essas mesmas mulheres, se a Lei de Assistência à Saúde fosse aprovada (Prickett; Angel, 2011).

Por fim, até então, consideramos que uma pesquisa começa com dados de nível individual. Contudo, este nem sempre é o caso, especialmente em estudos demográficos que podem analisar mudanças nas tendências populacionais ao longo do tempo ou entre coortes. Um conjunto de dados secundários de nível individual pode ser utilizado para 
observar variáveis mais detalhadas, não disponíveis em bases de dados de nível populacional, para explorar fatores que possam estar causando as tendências ou desigualdades expostas na análise. Identificar os possíveis mecanismos geradores de tendências não somente ajuda a localizar aqueles que poderão ser alvo de políticas, mas também orienta futuras pesquisas e coletas de dados (Olsen, 2004). Nos EUA, as minorias utilizam relativamente menos os asilos de idosos, ao envelhecer. Essa tendência poderia ser investigada por meio de bases de dados do tipo censitário, tais como o American Community Survey, de modo a identificar os fatores de incapacidade e de saúde que tornam os diferentes grupos raciais e étnicos suscetíveis de utilizar asilos, e como diferem essas trajetórias. Onde a mesmas divergem, estudos epidemiológicos menores, como o H-EPESE, que utilizem medidas mais complexas e minuciosas das condições de saúde, podem ser aproveitados para examinar por que razão as medições mais amplas e globais de incapacidade são menos eficientes para explicar tais desigualdades. Essa informação pode ser usada para orientar questões programáticas direcionadas à redução das diferenças no uso dos cuidados institucionais, ou para indicar que tipo de variáveis poderia ser somado aos esforços de coleta de dados em grande escala, de modo a aprimorar a identificação de desigualdades relacionadas à saúde e às necessidades especiais.

\section{Desafios e Perspectivas}

Apesar das oportunidades apresentadas pela triangulação, os sociólogos devem ter em conta uma série de desafios importantes. Por sua própria natureza, a triangulação utiliza uma variedade de fontes de dados que, em geral, ampliam a quantidade de dados disponíveis, bem como os marcos conceituais segundo os quais os dados foram coletados. É importante utilizar a triangulação tendo por base uma sólida análise ou método 
primário e, a partir daí, escolher um método secundário adequado para orientar a análise primária (Olsen, 2004). Hierarquizar os métodos ou análises propostos permite focar as questões de pesquisa. A triangulação, nesses termos, ajuda a contextualizar ou impulsionar resultados importantes embora estatisticamente fracos e se constitui em um meio para explorar achados incomuns e "erros sociológicos" em análises primárias.

Ao utilizar uma fonte de dados, os pesquisadores devem estar particularmente atentos a questões técnicas envolvidas na construção e coleta de dados das diferentes fontes. Quando estas envolvem distintas variáveis e marcos conceituais, é muito provável que a metodologia usada durante a coleta de dados e a própria construção da base de dados possam levar a uma série de vieses (Broege; Owens; Graesch; Arnold; Schneider, 2007). É importante ter em conta e conhecer as potenciais fontes de viés, mesmo dentro das mesmas populações e com a ponderação adequada. Por exemplo, o H-EPESE faz um levantamento dos americanos de origem mexicana dos quatro estados que fazem fronteira com o México e o Colorado e inclui entrevistados de 65 anos ou mais, enquanto o PUMS-O inclui entrevistados de 60 anos ou mais, e tem representatividade nacional. Assim, o viés, aqui, seria uma amostra de pessoas mais pobres e mais idosas (e, portanto, mais frágeis) em contraposição a uma amostra com um perfil comparativamente mais jovem e rico.

Em termos do uso da triangulação no contexto das políticas, os pesquisadores tendem a apoiar-se em fontes de dados menores, mais seletivas, e contextualizar os resultados com dados censitários mais abrangentes. Outra cilada comum (e uma cilada não exclusiva à triangulação, mas a todos os estudos que tentam generalizar resultados para padrões mais amplos de comportamento em nível populacional) é criar uma falácia ecológica. Por exemplo, a triangulação de fontes de dados poderia levar medições de nível individual a serem falsamente transpostas para ten- 
dências mais amplas em nível populacional. Ou, também, o oposto, quando subgrupos de pessoas são desagregados de bases de dados de nível individual originadas de características incomuns no nível populacional, e quando são feitas inferências sobre os mecanismos que impulsionam essas tendências, utilizando-se uma base de dados epidemiológicos, mais complexa.

Por fim, uma crítica teórica do uso de triangulação nas ciências sociais relembra uma das motivações originais por trás do uso deste método; fundamentalmente, a de que o processo de articulação complementar através da triangulação pode ajudar a produzir um quadro mais holístico e preciso das vidas sociais. Se, por um lado, a capacidade de integrar metodologias entre diferentes disciplinas e marcos conceituais tem sido uma virtude da triangulação, ela também constitui seu ponto fraco, pois envolve certa desconsideração das diferentes premissas epistemológicas e ontológicas que são necessárias ao coletarem-se dados sob a perspectiva de diversas disciplinas (Blaikie, 1991).

\section{Conclusão}

Neste artigo, exploramos como a triangulação pode ser usada para analisar grupos populacionais constituídos por minorias vulneráveis, com vistas a obter uma representação mais abrangente de suas vidas. Discutimos a triangulação utilizando exemplos de diversas bases de dados relevantes dos EUA, as quais investigam a população idosa de origem latino-americana, com foco na interseção entre as condições de saúde e incapacidades e os apoios sociais.

Argumentamos que a triangulação pode não apenas replicar e reforçar padrões a partir de amostras pequenas, produzindo resultados mais robustos, mas que, também, pode ser utilizada para traduzir achados sociológicos para o contexto das políticas. Por exemplo, a triangulação 
pode problematizar questões de pesquisa para formuladores de políticas ou transformar achados de nível individual em tendências de nível populacional. Além disso, a triangulação pode ser usada para influenciar prioridades políticas, inclusive as prioridades de futuras pesquisas e coletas de dados.

Por sua própria natureza, a sociologia conta com um amplo espectro de marcos conceituais e teorias sobre os quais se baseia, o que a torna um campo apto ao uso da triangulação. O campo deveria se aproveitar desta flexibilidade conceitual e metodológica para tornar suas descobertas acessíveis a, e aplicáveis por formuladores de políticas. Isso pode assegurar não só que a sociologia se constitua em um campo relevante para futuros financiamentos de pesquisa, mas também, o que é mais importante, assegurar uma melhor compreensão das complexidades da vida social de populações e, especialmente, de populações minoritárias e de difícil alcance, bem como clareza nas decisões e desdobramentos de políticas.

\section{Referências}

1. ALTSHULER, Norma; SCHIMMEL, Jody. Aging in Place: Do Older Americans Act Title III Services Reach Those Most Likely to Enter Nursing Homes? Washington, DC: Mathematica Policy Research Reports, 2010. (Issue Brief, Number 1).

2. ANGEL, Jacqueline L.; HERRERA, Angelica; MARKIDES, Kyriakos; TORRESGILL, Fernando. Can older Mexican-Americans age in place? The nexus between family and community-based long-term care. In: Cerontological Society of America Meeting. 2011. Boston, Massachusetts.

3. ANGEL, Jacqueline L.; HOGAN, Dennis P. The Demography of Minority Aging Populations. Journal of Family History v. 17, n. 1, p. 95-115, 1992.

4. ANGEL, Jacqueline L.; MONTEZ, Jennifer Karas; ANGEL, Ronald J. A Window of Vulnerability: Health Insurance Coverage among Women 55 to 64 Years of Age. Women's Health Issues, v. 21, n. 1, p. 6-11, 2011.

5. BLAIKIE, Norman W. H. A critique of the use of triangulation in social research. Quality \& Quantity, v. 25, n. 2, p. 115-136, 1991. 
6. BROEGE, Nora; OWENS, Ann; GRAESCH, Anthony P.; ARNOLD, Jeanne E.; SCHNEIDER, Barbara. Calibrating Measures of Family Activities Between Largeand Small-Scale Data Sets. Sociological Methodology v. 37, n. 1, p. 119-149, 2007.

7. DAVIS, Karen. National survey of Hispanic elderly people, 1988. Ann Arbor, MI: Inter-university Consortium for Political and Social Science. 1990. v. 2.

8. DENZIN, Neil K. The research act: A theoretical introduction to sociological methods. 2nd. ed. New York: McGraw-Hill. 1978.

9. MARKIDES, Kyriakos S.; RUDKIN, Laura; ANGEL, Ronald J.; ESPINO, David V. Health status of Hispanic elderly. In: MARTIN, Linda G.; SOLDO, Beth J. (Eds.) Racial and Ethnic Differences in the Health of Older Americans. Washington, DC: National Academy Press, 1997.

10. OLSEN, Wendy K. Triangulation in social research: Qualitative and quantitative methods can really be mixed. In: HOLBORN, M.; HARALAMBOS, M. (Eds.) Developments in Sociology. Ormskirk: Caseway Press, 2004.

11. PALLONI, A.; ARIAS, E. Paradox Lost: Explain the Hispanic Adult Mortality Advantage. Demography, v. 41, n. 3, p. 385-415, 2004.

12. PRICKETT, Kate C.; ANGEL, Jacqueline L.. The New Health Care Law: How Will Women Near Retirement Fare? Women's Health Issues, v. 21, n. 5, p. 331337, 2011.

13. SMELSER, Neil J. The Optimum Scope of Sociology. In: BIERSTEDT, Robert. (Ed.) A Design for Sociology: Scope, Objectives, and Methods. The American Academy of Political and Social Sciences, Philadelphia. 1969.

14. WESTAT, Inc. A survey of elderly Hispanics: Final report. Rockville, MD: Westat, Inc., 1989.

Recebido em: 10/05/2012

Aceite final: 19/08/2012 